Cinémas

Revue d'études cinématographiques

Journal of Film Studies

\title{
La « vraie-res-semblance » au cinéma
}

\section{Lucie Roy}

Volume 10, numéro 1, automne 1999

\section{Cinélekta 3}

URI : https://id.erudit.org/iderudit/024806ar

DOI : https://doi.org/10.7202/024806ar

Aller au sommaire du numéro

Éditeur(s)

Cinémas

ISSN

1181-6945 (imprimé)

1705-6500 (numérique)

Découvrir la revue

Citer cet article

Roy, L. (1999). La « vraie-res-semblance » au cinéma. Cinémas, 10(1), 111-129. https://doi.org/10.7202/024806ar

\section{Résumé de l'article}

L'auteure fait cette supposition que la vraisemblance est tenue dans les rets de la perception d'image. Dans cet esprit, il importe, croit-elle, que soient élucidés " [...] le langage [filmique] et la nature de sa relation [perceptive], "réaliste", avec la réalité [...] » (Nordahl Lund, 1992, p. 65). Bien que le travail ici mené ait un caractère interrogatif, réflexif, l'auteure se prête, sous l'angle de la perception et, conséquemment, de la vraisemblance, à l'analyse de deux films, dits documentaire et fictionnel, qui portent sur un même sujet : l'intervention de Schindler pour la survie des Juifs victimes de l'Holocauste. 


\title{
La «vraie-res-semblance» au cinéma ${ }^{1}$
}

\section{Lucie Roy}

\section{RÉSUMÉ}

Lauteure fait cette supposition que la vraisemblance est tenue dans les rets de la perception d'image. Dans cet esprit, il importe, croit-elle, que soient élucidés «[...] le langage [filmique] et la nature de sa relation [perceptive], "réaliste", avec la réalité [...]" (Nordahl Lund, 1992, p. 65). Bien que le travail ici mené ait un caractère interrogatif, réflexif, l'aureure se prête, sous l'angle de la perception et, conséquemment, de la vraisemblance, à l'analyse de deux films, dits documentaire et fictionnel, qui portent sur un même sujet: l'intervention de Schindler pour la survie des Juifs victimes de l'Holocauste.

\begin{abstract}
The author postulates that versimilitude is bound to the perception included in the image. Accordingly, it does matter, she believes, that "[...] [filmic] language and the nature of its 'realistic' [perceptual] relation to reality" (Nordahl Lund, 1992: 65) be clarified. Despite the interrogative and reflexive nature of this work, the author sets out to analyze two films, one documentary and the other fictional, both on the same subject: Schindler's intervention resulting in the rescue of Jews from the Holocaust. She does so under the angle of perception, and therefore of verisimilitude.
\end{abstract}

Le monde est langage et parole.

Ludwika Mostowicz, "Vers la phénoménologie sémiotique ${ }^{2}$ " 


\section{L'image en mouvement qui fixe}

Inspirée par le champ d'investigation de la phénoménologie, je tenterai de mettre en évidence ce que je tiens actuellement pour les grands principes ${ }^{3}$ de la perception filmique et $j$ 'aborderai, à partir de là, la problématique de la vraisemblance au cinéma.

Je ferai tout d'abord remarquer que l'emploi du terme "problématique» associé à la vraisemblance témoigne par avance de son caractère changeant et que l'association que je fais entre un champ d'études cinématographiques et la "perception d'image " ", qui est, elle, moins souvent appliquée à l'étude du cinéma, exige l'exercice d'une pensée de l'exploration, d'une pensée qui soit, je dirais, plus rêveuse qu'affirmative. En effet, si, par habitude presque, entrent souvent en jeu les questions relatives à la vraisemblance dans le cadre des études cinématographiques, est, à propos de cette vraisemblance, moins usuel l'examen de la qualité d'être de l'image qu'est l'iconicité qui, telle que je la considère, se manifeste précisément par la perception.

Donc, inspiré par une "discipline douce» — selon la belle expression de Jean Fisette (p. 16) - ce texte devrait laisser apparaître les perpétuels croisements que l'appareil filmique signale entre, à l'aller (du côté de l'écriture), une phénoménologie présente sous la forme de la perception et l'appareil sémiosique du film, comme entre, au retour (du côté de la lecture), la vraisemblance, qui a trait aux procédés systémiques liés à l'appareil sémiosique du film, et la compréhension, phénoménologique je dirais, des mêmes procédés systémiques et ce, en rapport avec tous les autres qui meublent l'univers de la lecture.

Si, dans le cadre de la petite partition des motifs portés à l'étude que je viens de faire, je semble éviter quelque peu la question de l'iconicité précédemment associée à la qualité d'être de l'image, c'est que je tiens l'iconicité pour un signe, le signe relatif de ces perpétuels croisements entre, comme je le supposais à l'instant, une perception (phénoménologique et sémiosique) et une vraisemblance (sémiosique et phénoménologique). Comme, en effet, je prétends devoir partir de là, de cette idée de l'iconicité, pour discuter de la perception et de son rapport à la vraisemblance, il m'apparaît quelque peu superflu de ne parler 
que d'elle, mais nécessaire d'examiner ce que, à leur propos, elle peut bien raconter.

\section{Au regard de la perception}

Je citerai tout d'abord une phrase de Wittgenstein qui me semble exemplaire et par sa simplicité et par la percutante compréhension de la perception non pas systémique mais phénoménologique qu'elle facilite:

Le champ visuel n'a pas en fait une telle forme:

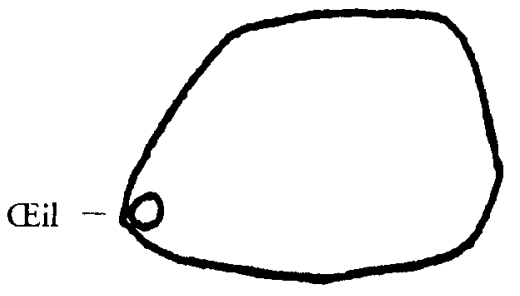

(Wittgenstein, p. 94).

Parce que le cinéma est le lieu d'une perception "pro-jetée», le lieu, en conséquence, d'une perception systémique, le champ visuel au cinéma n'a pas davantage cette forme.

Or, si le champ visuel du cinéma n'a pas cette forme, il n'a pas non plus, me semble-t-il, cette autre forme, celle que propose Gardies en page 30 de son ouvrage L'Espace au cinéma:

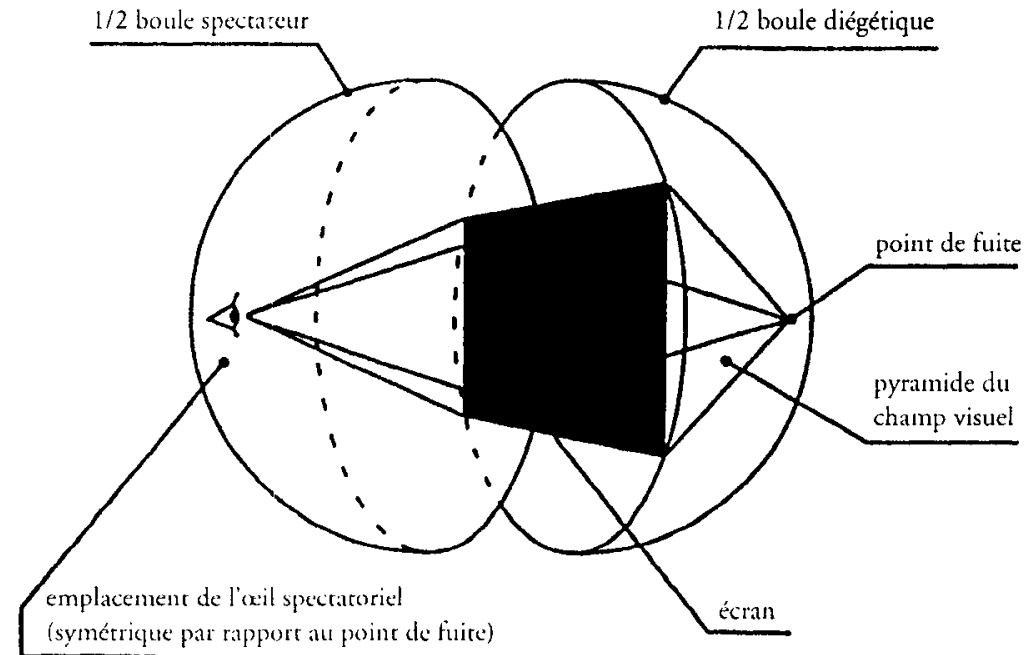


Je propose plutôt de remplacer l'œil spectatoriel, contenu dans cette boule spéculaire proposée par Gardies, par un œil phénoménologique / sémiosique, c'est-à-dire une perception qui, conservant son caractère phénoménologique, met en œuvre, "ouvrage" en quelque sorte, espace et temps au cinéma.

Je le fais en espérant par la même occasion exclure toutes les références relatives à l'identification cinématographique primaire et afin d'insister sur l'assertion que toute perception d'image au cinéma est le fait d'une perception "pro-jetée».

"Lidentification primaire, au cinéma, c'est, je le rappelle, celle par laquelle le spectateur s'identifie à son propre regard et s'éprouve comme foyer de la représentation, comme sujet privilégié, central et transcendantal de la vision" (Aumont et al., p. 185). Nuançant ce genre de propos, qui tel quel me semble approximatif parce que trop entier, je relève cette évidence, et une évidence qui, loin d'être assortie à une réflexion psychanalytique sur le cinéma comme c'était le cas plus tôt, est, j'insiste là-dessus, associée à un projet plus inspiré par la phénoménologie, c'est-àdire pour le cas sémiotique qui m’occupe, un projet perceptif: pour voir à l'écran, il faut bien emprunter le sentier du regard dessiné par l'œil phénoménologique qui configure les espaces de l'écran, il faut contempler les dessins phénoménologiques que la perception produit. Quant à la lecture des "scènes sémiosiques" de l'écran, c'est, en effet, le seul centre d'action de la perception qui soit disponible, la seule phénoménologie susceptible d'être "lue» par le spectateur ou, si l'on préfere, la seule pragmatique du regard ou de l'écriture qui soit lisible.

Par ailleurs, l'expression "scène sémiosique " évoque très précisément, dans mon esprit, l'idée d'une scène. Sous l'angle de la perception, l'expression scène sémiosique désigne l'idée d'un lieu où serait langagièrement en jeu la perception. La perception langagière concerne ici l'image, la langue de l'image, mais sans que lui soit aussitôt associée son fait analogique; sans donc considérer aussitôt ce que l'image répète du monde. Aussi, si cette dernière précision tend à laisser entendre que la vraisemblance est plus ou moins tenue dans les rets de la perception, elle justifie, par la même occasion, le croisement de ces deux approches. 
Puisque à propos de la vraisemblance sont habituellement en jeu les battements référentiels impliqués par les systèmes et puisqu'elle se trouve, comme je viens de le dire, dans les rets de la perception d'image, l'étude de la vraisemblance, sous l'angle des "scènes sémiosiques", exige, pour ainsi dire doublement, que soient élucidés " $[\ldots]$ le langage et la nature de sa relation "réaliste" avec la réalité [...]" (Nordahl Lund, p. 65). Le travail ici mené a un caractère interrogatif, réflexif.

Je parle ici de relation et non du retour de la réalité dans le langage, la langue de l'image. Le langage n'opère pas sur la seule reconnaissance du un à un, du un ici, dans la réalité ou le monde, à un, là, dans l'image.

L'existence idéale est fondée sur le document, non sans doute comme objet physique, non pas même comme porteur des significations une à une que lui assignent les conventions de la langue dans laquelle il est écrit, mais sur lui en rant que, par une "transgression intentionnelle" encore, il sollicite et fait converger toutes les voies connatssantes et à ce titre instaure et restaure un "Logos" du monde culturel (MerleauPonty, cité dans Mostowicz, p. 41-42).

Toute perception d'image, toute perception, autrement dit, comprise dans les images du film est, comme je l'avançais au début du présent texte, le fait d'une perception "pro-jetée", c'est-à-dire d'une "transgression" faite sur la belle enveloppe de la réalité (transgression mémorielle et imaginante il va sans dire), d'une interruption de son cours (bref, d'une perception intentionnelle qui, rétentionnelle, retient dans le lieu de l'image du temps et de l'espace ou qui, protentionnelle, appelle ou convoque à même le lieu de l'image le temps et l'espace environnant). D'où il apparaît que l'image parle du monde aussi bien par ce qu'elle ne montre pas que par ce qu'elle montre. Sous le joug de la rétention et de la protention qu'elle subit, "[...] l'image [même arrêtée, l'est] sur l'impossibilité de son arrêt" (Ropars-Wuilleumier, p. 45). Comme, en effet, chacun sait le sort rétentionnel et protentionnel qui lui est réservé, elle instaure et restaure aussi parfois, "vraisemblablement" je dirais, le logos du monde culturel: elle fait précisément appel à la 
raison - irraisonnée même — du monde culturel pour être comprise.

Bref, l'image, la perception d'image inscrite dans les scènes sémiosiques (la perception d'image sert, comme on l'aura compris, de révélateur à l'autre, à l'image) est "phénoménante", c'est-à-dire que, transgressive, elle instaure un monde ou restaure plus ou moins le monde, et cela en vertu précisément du sens mouvant du monde. C'est de la mouvance du monde dont elle témoigne. C'est dans cette mouvance qu'elle se tient; elle encore qu'elle retient.

Ainsi, si je choisis de reprendre à mon profit le terme «restauré » proposé par Merleau-Ponty, c'est pour tout d'abord impliquer l'événementialité représentationnelle de l'image: une image, en effet, intervient comme il a été dit sur le cours du monde et signifie de la sorte, mais en se signifiant tout d'abord elle-même; et c'est pour impliquer ensuite l'événement représentatif inclus dans l'image: une image signifie, comme on le suppose, autre chose qu'elle-même. Enfin, si je choisis de reprendre à mon profit le terme "restauré", c'est pour raconter un peu plus le signe même de son iconicité, c'est-à-dire: a) la singularité représentationnelle dont je viens de parler; b) la continuité de la réalité que l'image ou, mieux, que la perception d'image instaure, car c'est par la perception d'image que l'image se rappelle à la perpétuelle mobilité du monde - ce qui, par ailleurs, faisait dire à Deleuze que toute image est une imagemouvement.

Ainsi, si on peut reconnaître une infinie sémiosis relativement à l'interprétation, force est de constater l'infinie sémiosis que l'écriture appelle, c'est-à-dire - j'insiste sur ce terme — comprend. C'est dans cet esprit, sous l'angle de l'écriture filmique et non sous celui de la lecture d'un unique fait filmique, que j'entreprends d'aborder les questions de la vraisemblance au cinéma, dans cet esprit, aussi, que j'emploie le terme «sémiosis».

\section{Au regard de la perception (suite) et de son differement (première partie)}

Paraphrasant Wittgenstein donc, j'ose prétendre que le champ visuel du cinéma a une telle forme; une forme où la 
perception croise la cognition, où la perception ouvre à la cognition, la manifeste.

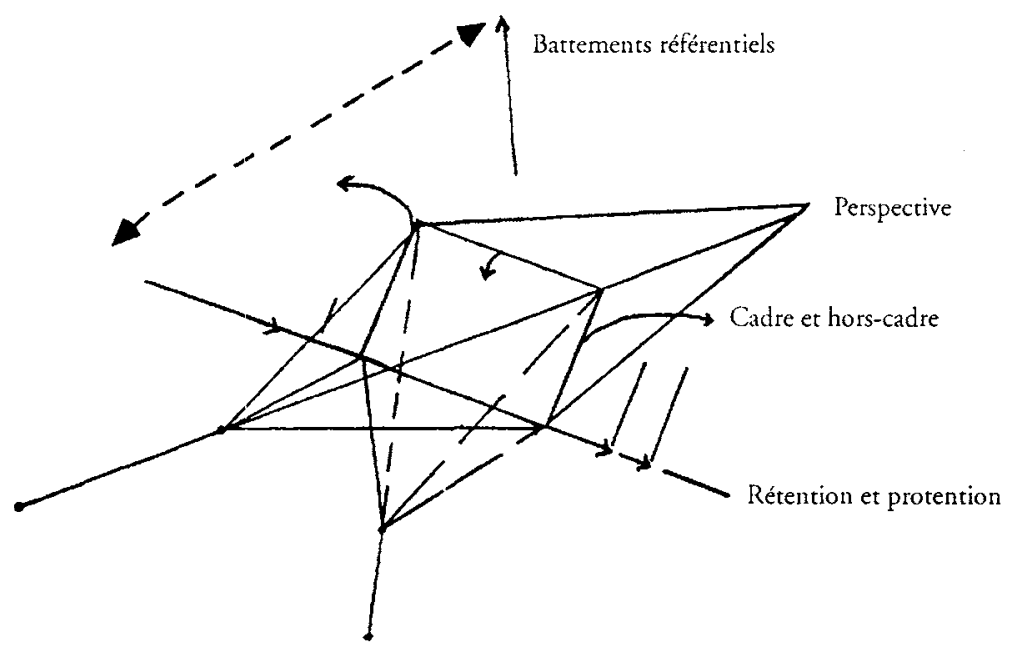

\section{- PERCEPTION PHÉNOMÉNOLOGIQUE / SÉMIOSIQUE}

Entre les deux, entre une scène phénoménologique telle que décrite a contrario par Wittgenstein et une scène sémiosique telle que je l'envisage, il y a de commun la perception, une perception qui, par "differement", agit différemment selon qu'il s'agit plutôt de représenter, en termes bergsoniens, la perception des choses (la "matière", le "réel" si l'on veut) ou qu'il s'agit plutôt de représenter des choses de la perception (la «mémoire», l'imagination).

Le principe du diffèrement, s'il semble s'inscrire en faux contre le dualisme qui, par automatisme sans doute, prévaut encore - même depuis Bergson - au sujet des choses et de la représentation, il le fait aussi, en un certain sens, au sujet des genres, qui opposent, là aussi presque automatiquement, le documentaire er la fiction. Documentaire pour lequel - d'une façon toute paradoxale - serait idéalisé le passage de la réalité à l'écran. Fiction pour laquelle - par un faux idéal sans doute on tendrait à ne reconnaître que dans une faible mesure le retou" de la réalité à l'écran. 
Le terme "differement» aide à explorer l'idée d'une perception d'image - ou la perception de l'"idée d'image " : une perception reconnue, au cinéma par exemple, contient une idée, une intentionnalité, sous la forme d'une image.

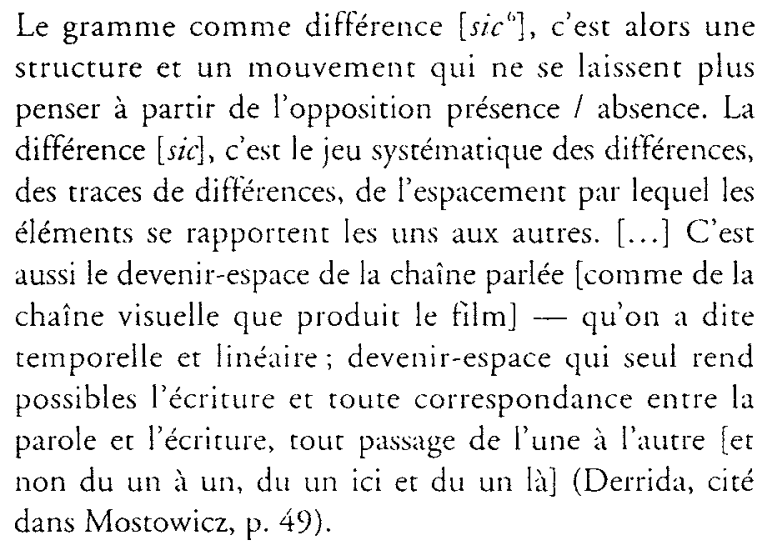

Donc le principe du differement dont je parle fait valoir l'idée que la perception est au cour des scènes sémiosiques du cinéma et qu'elle est par elle-même différentiellement significative. Car c'est elle, la perception, qui forme la structure et trace le mouvement. Ainsi, le differement à l'œuvre, en terme de perception, dans les scènes sémiosiques du film, fait le jeu systématique des différences, trace les différences, l'espacement par lequel des éléments se rapportent les uns aux autres. C'est le devenirespace, comme le devenir-temps, de la chaîne visuelle que produit le film.

\section{Du differrement (deuxième partie)... et de l'impossible arrêt sur l'image}

Sous l'angle des scènes phénoménologiques, on admet généralement que la perception de l'espace précède, en quelque sorte, sa cognition - je n'insisterai pas sur le retour de la mémoire par la perception, lequel en réfere, comme on le sait, à une autre phénoménologie que celle, sémiotique, à laquelle je me prête. À l'opposé, sous l'angle de scènes sémiosiques, on admet généralement le fait que la cognition précède la perception; il faut, de fait, (conce) voir avant de faire voir. 
Autour des deux scènes, donc, phénoménologique et sémiosique, se trouve, comme je le suppose, une sorte de noud: la perception, c'est celle qui, phénoménologiquement, voit et, selon l'exemple bergsonien, se souvient, et c'est celle qui, sémiosiquement, fait voir et, faisant voir, fait se souvenir et imaginer. Et c'est celle qui fait voir, par differement, différemment selon qu'il s'agit de figurer, par la perception, des états de monde réels ou, toujours par la perception, des états de monde fictionnels.

Je dis "états de monde réels et états de monde fictionnels" en imposant aux prédicats "réels" et "fictionnels" le pluriel pour préciser que, sur le plan, je dirais, de la célébration discursive se lisent continûment des états perceptifs et, conséquemment au cinéma, descriptifs du monde; des états qui peuvent être représentatifs de moments "du» monde, celui qui est dit réel (et dont font partie les événements et les faits du monde) et des états "de" monde, ceux qui sont dits fictionnels (et dont font partie les univers inventés). Dans la perspective qui m’occupe, qui comprend le sens - phénoménologiquement - mouvant du monde que convoque la perception, tout état "de" monde constitue un état "du» monde. Parce que les états de monde réels ou fictionnels sont pensés, parce que ces états sont réalisés par les textes, écrits et lus, tout état "de» monde constituerait un état "du» monde.

Or, je le précise, tout état n'est état que par recours à la représentation où agit le principe du diffèrement - on se souviendra, à ce propos, de la disctinction que j'ai faite relativement à l'événementialité représentationnelle et représentative, et on constatera, en vertu du lien que je fais ici, que toute représentation rend "réellement " compte d'un état qui, réel ou fictif, est à la fois représentationnel, présent par lui-même, et représentatif, présent pour quelque chose d'autre.

Donc, par leur représentation, c'est-à-dire le diffèrement relatif de leur représentation, les textes diraient leur appartenance à un autre état et en parleraient, parleraient d'un état du monde réel (même rêvé ou imaginé) ou d'un état du monde fictionnel (même gorgé de références au réel).

Ainsi, si je dis perception, j'implique aussitôt le mouvement de la perception; si je dis perception d'image et, conséquemment, mouvement de la perception placé dans l'espace de l'image, je dis 
aussi "arrêt" sur l'ordre mouvant du monde et, donc, relais, rappel dans l'image de l'ordre mouvant du monde; si je dis ordre, je veux dire mise en ordre, configuration du monde qui appelle, comme le fait la perception pour l'image, une idée. Si je dis idée, je dis intentionnalité et si, enfin, je dis intentionnalité, j’appelle différentiellement toutes les autres intentionnalités qui ne sont pas elle et par devers lesquelles celle-ci s'écrit. Si je discute du terme "perception" et de son principe, le differement, j'ajoute une réflexion ayant trait à son fait "phénoménant", c'est-à-dire au retour $\mathrm{du}$ monde dans les états représentationnels; retour $\mathrm{du}$ monde qui, pensé dans le langage et par le langage, ne participe que d' "une relation réaliste avec la réalité ".

\section{Deux suppositions, deux exemples et deux explications - DEUX SUPPOSITIONS}

Ainsi, par diffèrement justement, on supposera (c'est la première supposition) que, pour représenter la matière, le réel, des états de monde réels, la perception fixe et se fixe précisément sur les choses du réel: c'est le réel qui, par la perception, "est réalisé ». Et on supposera aussi (c'est la seconde supposition) que pour représenter des états de monde fictionnels, même gorgés de références au réel, la perception est susceptible d'utiliser la réalité en la "surfaçant", c'est-à-dire en l'utilisant comme une surface, en la mobilisant, en lui imposant un mouvement qui n'est pas son fait.

\section{- UN EXEMPLE}

Dans le film documentaire intitulé La Vraie Histoire d'Oskar Schindler (Jon Blair, 1994), par exemple, des survivants de l'Holocauste qui ont été aidés par Schindler prennent tour à tour la parole et témoignent de sa bonté. Une caméra, fixe, les prend en plan épaule ou poitrine tandis qu'ils regardent aux bords, droit ou gauche, de l'écran. Les survivants s'adressent visiblement à un intervieweur placé là. À cause de cela, on a l' impression" d'une relative disparition du dispositif de la perception d'origine cinétique, phénoménologique / sémiosique en fait, dans lequel le spectateur s'engage forcément. À cause de cela aussi, on induit une relation entre l'intervieweur et l'inter- 
viewé, intervieweur toujours hors cadre, mais convoqué dans le cadre par le regard de l'interviewé.

\section{- UNE EXPLICATION REIATIVE AU PREMIER EXEMPLE}

Bien que fixes, les images sont "[...] arrêtée[s] sur l'impossibilité de [leur] arrêt" (Ropars-Wuilleumier, p. 45), car si ce genre d'images et si ce genre de scènes sémiosiques nécessairement induites par ces images servent à faire passer le passé, elles aident aussi à penser le cadre comme une frontière, celle qui, précisément, permet, plutôt qu'elle n'interdit, le passage dans le cadre du hors-cadre, ne serait-ce que par le fait qu'il en laisse suspecter la présence. L'image est, plus particulièrement ici, arrêtée "sur l'impossibilité de son arrêt".

Bref, ce type d'images fixes, de perception d'images qui se fixe sur les témoins, joue le rôle de "tenant-lieu». Ce type d'image participe d'une relation réaliste et avec la réalité historique et avec la réalité du langage, et cela en vertu des rapports ou, mieux, des relais établis entre: a) intervieweur et interviewé; b) cadre et hors-cadre; c) temps actuel et temps passé; d) perception phénoménologique et relais qui ont précisément servi à plus ou moins l'occulter.

Je ferai remarquer l'ambiguïté relative du lieu en principe occupé par le spectateur. Comme en effet l'interviewé regarde du côté de l'intervieweur, comme la perception phénoménologique/sémiosique fournie dans l'espace de l'image occupe une position différente, on se demande quel lieu ou quelle autre position peut bien imaginairement occuper le spectateur. Quel autre lieu que, pour ainsi dire, le fauteuil du milieu ${ }^{8}$.

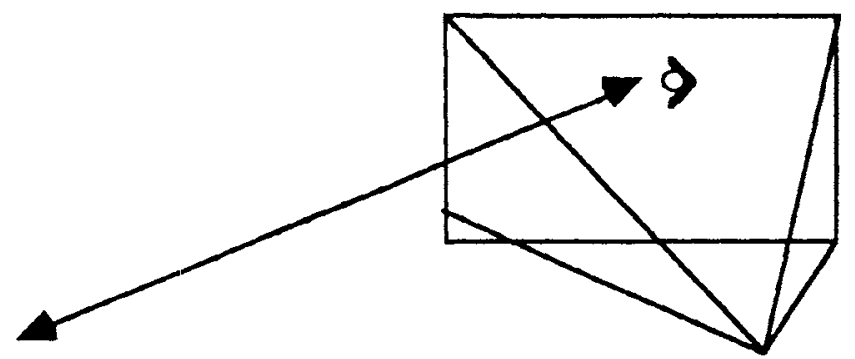

intervieweur

perception phénoménologique/sémiosique 


\section{- RETOUR SUR LA SUPPOSITION}

Et, on supposera aussi que pour rendre vraisemblable la fiction à l'écran, la perception se fait mouvement. C'est du moins ce que, en d'autres termes et dans un autre esprit, faisait déjà remarquer Metz dans son chapitre intitulé "À propos de l'impression de réalité au cinéma" :

Le mouvement apporte donc deux choses avec lui : un indice de réalité supplémentaire et la corporalité des objets. [...] le mouvement contribue à l'impression de réalité de façon indirecte (en donnant du corps aux objets), mais il y contribue aussi de façon directe, pour autant qu'il apparaît lui-même comme un mouvement réel. C'est en effet une loi générale de la psychologie que le mouvement, à partir du moment où il est perçu, est le plus souvent perçu comme réel, contrairement à bien d'autres structures visuelles telles que le "volume" par exemple, qui pour sa part peut fort bien être perçu comme irréel lors même qu'il est perçu [...] (p. 17).

En effet, si l'on peut douter, en termes de représentation, de l'existence véritable des choses surfacées à l'écran, on ne saurait douter de l'existence du mouvement de la perception, mouvement et perception portés sur ces choses. Je note, au surplus, que lorsque la perception se fait mouvement, lorsque, pour le dire autrement, le mouvement fait "être" ces choses de la perception, la perception "est". C'est ce "est" de la perception qui justifie le principe du differement que je fais ici intervenir, car c'est ce "est " de la perception qui, à l'écran, fait "advenir" les choses.

\section{- LE DEUXIÈME EXEMPLE}

La comparaison que je fais, à l'emporte-pièce il faut le dire? entre la caméra qui fixe et celle qui se manifeste par le mouvement, a, en fait, pour référence deux films qui s'intitulent La Vraie Histoire d'Oskar Schindler et La Liste de Schindler (Steven Spielberg, 1993).

Le premier est un documentaire, pour lequel j'ai décrit l'usuelle figure qui implique, par fixité, un relais d'intervieweurs à interviewés, notamment. Le second, fiction à caractère 
historique, fait, lui, perpétuellement appel à la mobilité de la caméra qui explore des lieux et des actions qui ont fait l'Histoire. C'est, par exemple, le camp de Cracovie et c'est, à l'intérieur de ce dernier, la dernière rafle dont les Juifs sont victimes. Perpétuellement, la caméra, alternativement sur trépied et à l'épaule, opère par avance et recul. Elle participe d'une perception en action ${ }^{10}$.

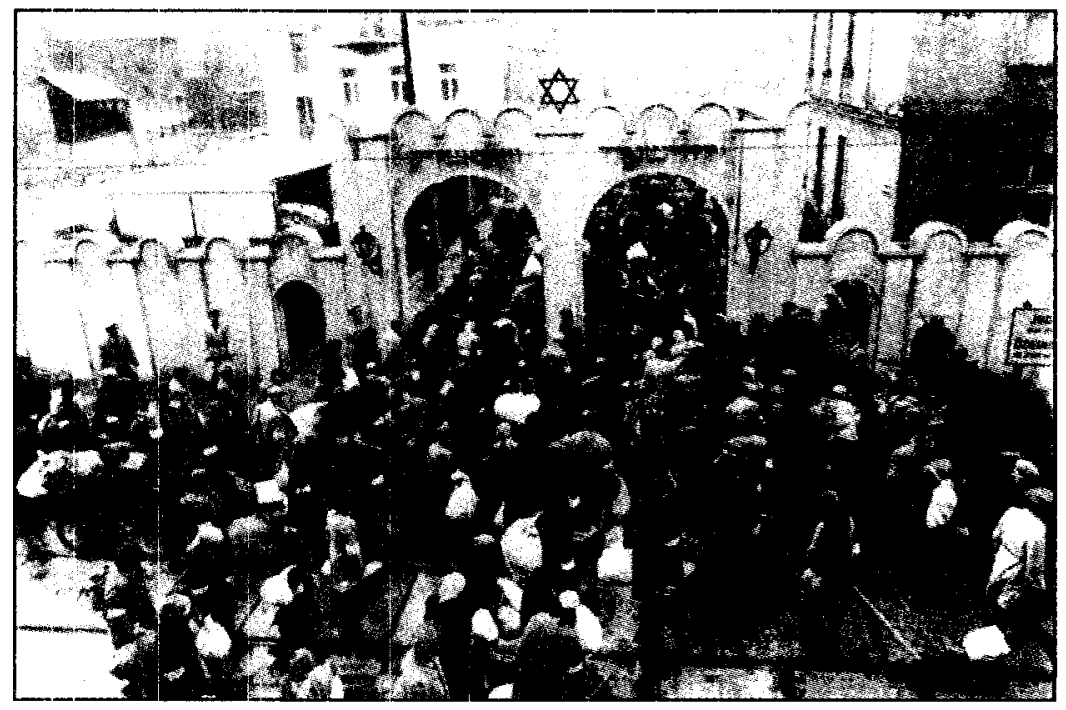

\section{La Liste de Schindler (Steven Spielberg, 1993)}

\section{- RELATIVEMENT AUX PREMIER ET DEUXIEME EXEMPLES}

Ces dernières observations font ressortir l'idée que, relativement au premier exemple: a) l'image qui fixe les choses de la représentation, c'est-à-dire l'image "tenant-lieu ", est susceptible de recouvrer, par évocation et à cause de l'invisibilité qui agit hors du cadre mais que le cadre rappelle, son plein pouvoir de mouvement, de mise en mouvement du temps et de l'espace et, conséquemment, ses pleines possibilités d'ekphrasis (l'image, aije rappelé plutôt, y est, tel qu’y songeait Ropars, arrêtée dans l'impossiblité de son arrêt) et que, à l'inverse, relativement au deuxième exemple, b) l'image suturée par le mouvement, si elle 
participe de l'ekphrasis, ne rappelle pas ultimement le tissu de l'invisibilité, d'où, somme toute, l'image aurait pu venir s'il s'était agi de montrer la réalité même et en son temps; l'image, ici, est le lieu, un lieu gorgé toutefois de réalité, perceptive d'abord, historique ensuite.

\section{La vraie-res-semblance ${ }^{11}$}

J'entreprendrai, dans la seconde partie de ce texte, une réflexion portant plus directement sur le rapport de la perception d'image au vraisemblable, perception ou mouvement d'une perception-action, d'une perception "en" action qui, même immobile, non seulement se prête au jeu de la vraisemblance mais, tel que le suppose en fait véritablement le jeu, la produit.

Avant d'aborder de front cette question de la perceptionaction en rapport avec la vraisemblance, un détour me semble nécessaire. Je dis "détour", j'aurais aussi bien pu dire une explication supplémentaire me paraît à propos, car j'ai signalé sans l'expliquer davantage le retour à l'opposition, bien connue depuis Bergson, entre matière, chose (ce que l'on tient pour le référent en termes sémiologiques) et mémoire, représentation (ce qui équivaut à la référence, aux tracés référentiels en présence dans les récits). Cette opposition - provisoire chez Bergson -, j'aimerais l'associer à une supposition qu'a faite Steffen Nordahl Lund. Car, si apparaît, chez Bergson, une opposition et, aussitôt, une sorte de chassé-croisé entre la matière et la mémoire, se reconnaît ailleurs, dans les propos de Nordahl Lund notamment, un croisement entre la matière textuelle (le récit et son pseudo-référent) et la mémoire des textes et les façons de les écrire (le code extra-diégétique si l'on veut).

Donc Nordahl Lund considère, et il n'est évidemment pas le seul, que "[...] l'effet de réalité est bien ainsi l'effort d'authentification, moins du récit lui-même et de son pseudo-référent que du code extra-diégétique de sa référence. Seulement, ce réel référencié n'est lui-même que d'effet» (p. 65).

Il mapparaît tout à fait opportun de tenir compre du code extra-diégétique de la référence d'où, par effort d'authentification, proviennent l'effet de réalité, un réel qui, ainsi référencié, 
n'est que d'effet. Les propos de Nordahl Lund témoignent pertinemment d'une relation, réaliste ou non — vraisemblable ou non —, avec la réalité et le langage, d'une relation avec, je le souligne, la réalité du langage.

En résumé, sous le rapport de la vraisemblance, la perception ne recouvre pas seulement l'aspect spatio-temporel, la géographie des images - filmiques et littéraires —, mais également une autre géographie, textuelle celle-là, que forme l'ensemble des langages, des récits, des écrits.

Dans la perspective qui m'occupe, je puis donc supposer que c'est le lien entre le filmeur et le filmé qui est, au regard de la perception, "pro-jeté" par le langage et dans les récits; ou, autrement dit, que la perception d'image et la scène sémiosique qu'elle fait naittre sont constitutives d'une autre scène, cognitive celle-là, dont dépend l'ode à la vraisemblance ou à l'invraisemblance. Autrement dit encore, de ce code extra-diégétique dépendent également des perceptions d'image, des cognitions d'image, des façons de filmer la réalité (même imaginée), de dire sa langue et son langage par l'image.

De quelque façon qu'on la considère, c'est bien elle tout d'abord, la perception, qui fait alterner choses du réel (matière) et choses de la représentation (mémoire); perception, donc, qui, en ses effets cognitifs, produit, configure l'image, le récit, la diégèse, le texte et, cela, sans que l'on puisse dire si ces choses se trouvent tout à fait dans le texte non plus que tout à fait hors du texte.

C'est dans certe perspective que je souhaitais, à pas lents, mettre en évidence les lieux de la "vrai-semblance" ou, mieux, de la "vraie-res-semblance». Ce "res", malgré le luxe terminologique qu'il implique, désigne ici précisément l'être, c'est-à-dire une perception qui est. Donc, cette lecture de la vraie-ressemblance concerne, bien sûr, l'iconicité des matériaux filmique - comme dans un esprit quelque peu ouelletien ${ }^{12}$ celle des matériaux littéraires -, dans la mesure où ils rendent compte d'une perception d'origine, d'une cognition d'origine.

Une dernière précision s'impose, toutefois, sur le rapport de la perception à la cognition phénoménologique et sur celui de la cognition à la perception sémiosique évoqués plus tôt : la 
perception sémiosique croise très précisément la cognition phénoménologique, elle en permet le rappel et le retour.

Perception, cognition : cette présence "est". " (Et nous saluons au passage, recyclée, la réalité que Husserl nommait "doxique" en tant qu'expérience du sens de l'être dans la formeprésence du "est")" (Nordahl Lund, p. 77).

Le vraisemblable tient lieu de réel, substitué à ce qui se dérobe sous lui et le rend possible comme médiateur de discours, c'est-à-dire comme à la fois principe et résultat, cause et effet: signifié ultime du discours qu'il aura organisé et orienté dans sa visée du monde. Il désigne le réel (là-bas, de l'autre côté), mais c'est pour être lui-même (ci-devant) ce réel désigné. Par-delà il n'y a rien, ou plutôt il n'y a rien d'autre que ce qui puisse être parlé, que ce, donc, qui pour cela se trouve déjà impliqué au départ dans la seule forme de la parole (Nordahl Lund, p. 77).

Bref, la "vraie-res-semblance" reposerait sur trois traits: a) sur ce qui, étant de l'ordre de la perception, est et est vraie (il s'agit, comme il a été dit, d'une présence dont on ne peut douter); b) sur ce qui, étant de l'ordre du differement dans les systèmes, connaît, reconnaît, fait connaître - sur, autrement dit, ce qui, étant de l'ordre du differement "est", et est en entretien avec les choses du langage et les choses du monde (res); c) sur ce qui, étant entre états de monde réels et états de monde filmiques, ressemble ou non aux choses, aux états du "réel ( "semblance").

\section{Conclusion non facultative}

Ainsi, dans les exemples filmiques cités, la vraisemblance vient du mouvement de la "perception d'image" comme de la "cognition d'image». Dans le documentaire La Vraie Histoire d'Oskar Schindler, on rive, en effet, le spectateur à un endroit de l'espace du film. Dans la docu-fiction ou la fiction documentée La Liste de Schindler et, spécifiquement, dans le passage du film que j'ai signalé, c'est le contraire, on le fait se déplacer inlassablement. Les personnages du film, "les corps environnants" (pour reprendre l'expression de Bergson) ne sont pas assurés contre l'action immédiate de l'image que représente le champ de 
la perception ${ }^{13}$; ici, au contraire, la menace est vive. La perception rend compte d'une impression de panique: on assassine! Cette perception d'image et, j'ajoute, cette cognition d'image sont, par une opération de differement, par elles-mêmes significatives, par elles-mêmes tenues pour vraisemblables, elles disent ce que l'Holocauste a d'infigurable - d'invraisemblable!

\section{Université Laval}

\section{NOTES}

1 Ce texte, largement remanié, est issu d'un colloque organisé en mai 1998 par Louis Hébert dans le cadre des activités de la Société de sémiotique du Québec. Accueilli par l'ACFAS, ce colloque a donné lieu à des discussions fort intéressantes. Je remercie Louis Hébert pour avoir ménagé ce lieu de rencontre et pour avoir généreusement commenté la proposition de communication que je lui avais d'adord adressée.

2 C'est moi qui souligne.

3 Je me permets de renvoyer le lecteur qui espérerait trouver ici une analyse filmique à la définition du terme "principe» : un principe sert à considérer une proposition posée et non déduite après coup, après, pour ce qui concerne la présente étude, l'examen d'un film particulier. Comme, "en principe", il n'y a pas d'interdiction relative à l'étude de l'écriture filmique, comme nous ne sommes pas tous tenus de nous plier à une même pratique de l'analyse du film et malgré le fait que la présente étude puisse paraître un peu lourde pour certains, je m'autorise cette démarche.

Je précise, enfin, que ma démarche n'est pas accidentelle: j'étais résolue à emprunter ce parcours. La première partie de mon travail est forcément plus théorique: j'y discute de ce que je considère relever des grands principes relatifs à la perception et à la vraisemblance. La seconde a, elle, davantage trait à une pratique de l'analyse qui, comme je le mentionne dans le présent texte, constitue la suite d'une autre analyse menée ailleurs, dans un premier article intitulé "L'image au cinéma ou le corps (d)écrit " (CiNéMAS, vol. 7, $\mathrm{n}^{\text {"w }} 1-2,1996$, p. 11-35). Bien que s'y trouve des considérations d'ordre théorique, la lecture de ce premier article risque de satisfaire davantage le lecteur avide d'analyse filmique.

4 Voir mon artiche, à paraître, et qui porte ce titre.

5 C'est cette expression que privilégiait Marie-Claire Ropars-Wuilleumier dans son ouvrage L'Idée d'image (Saint-Denis: Presses Universitaires de Vincennes, 1995).

6 On aurait dû lire «différance».

7 Je dois l'idée qui suit à une étudiante, Véronique Larouche-Filion, qui m'a autorisée à l'explorer dans le cadre du présent texte.

8 C'est, je crois bien, ce lieu qu'il occupe et non cet autre lieu, celui fourni par le foyer du regard et dont on dit qu'il est propice au jeu de l'identification cinématographique primaire.

9 Je dis à l'" emporte-pièce", car des explications manquent, des explications et des nuances.

10 Schindler et sa compagne arrivent à cheval et regardent, du haut d'un promontoire, les Juifs du ghetto qui font l'objet d'ure rafle de la part des soldats allemands. 
Une série de plans en alternance montrent les Juifs terrorisés, des soldats allemands les exécutant, les poussant, les repoussant, après les avoir "classés", après, donc, avoir "différencié" hommes, femmes et enfants.

Une caméra à l'épaule s'active, par avance et recul, en visant des personnages qui sont des corps à abattre. Vus de loin, puis de près, vus de près pour être vus de loin, puis de loin pour être vus de près, les plans montrant les Juifs se succèdent en renversant perpétuellement, de près en loin justement, les symétries initiales. Des Juifs meurent. Cette description provient d'un article dont je suis à l'origine. Comme je la crois bonne cette description, il m'apparaît inutile d'en reprendre la formulation. Voir "Limage au cinéma ou le corps (d)écrit (CiNéMAS, vol. 7, n"1-2, 1996, p. 24-25).

11 Le mot res contenu dans le terme vraie-res-semblance dont je me permets l'usage, est un mot latin et signifie "chose, objet, être, affaire, fait, événement, circonstance" (Félix Gaffiot, Dictionnaire illustré latin-français, Paris: Librairie Hachette, 1934, p. 1349). J'ajoute cette phrase de Merleau-Ponty, que j'associe à la vraisemblance, pour dire un peu plus la problématique du réel en lui-même et, dans un autre ordre d'idées, d'une perception du réel dont la description philosphique est toujours difficile: "Nous voyons les choses mêmes, le monde est cela que nous voyons: des formules de ce genre expriment une foi qui est commune à l'homme naturel et au philosophe dès qu'il ouvre les yeux, elles renvoient à une assise profonde d' opinions" muettes impliquées dans notre vie. Mais cette foi a ceci d'étrange que, si l'on cherche à l'articuler en thèse ou énoncé, si l'on se demande ce que c'est que nous, ce que c'est que voir et ce que c'est que chose ou monde, on entre dans un labyrinthe de diffcultés et de contradictions" (Le Visible et l'invisible, Paris: Gallimard, 1979, p. 17). La problématique relative au réel et à la perception du réel au cinéma est semblablement problématique sitôt, en effet, que l'on tente d'en rendre compte, de la décrire.

12 Voir l'ouvrage de Pierre Ouellet intitulé Voir et savoir. La perception des univers du discours (Candiac: Éditions Balzac, 1992).

13 «De fait, j'observe [dans ces scènes sémiosiques, dans ces images qui sont aussi de petits récits] que la dimension, la forme, la couleur même des objets extérieurs se modifient selon que mon corps s'en approche ou s'en éloigne, que la force des odeurs, l'intensité des sons, augmentent et diminuent la distance, enfin que cette distance elle-même représente surtout la mesure dans laquelle les corps environnants sont assurés, en quelque sorte, contrc l'action immédiate de mon corps" (Henri Bergson, Matière et mémoire, Paris: PUF / Quadrige, 1990, p. 15).

\section{OUVRAGES CITÉS}

Aumont, Jacques et al. L'Esthétique du film. Paris: Nathan, 1983.

Bergson, Henri. Matière et mémoire. Essai sur la relation du corps à l'esprit. Paris: PUF/ Quadrige, 1990.

Fisette, Jean. Pour une pragmatique de La signification. Montréal: XYZ éditeur, 1996. Gaffiot, Félix. Dictionnaire illustré latin-françats. Paris: Librairie Hachette, 1934.

Gardies, André. L'Espace au cinéma. Paris: Méridiens Klincksieck, 1993.

Merleau-Ponty, Maurice. Le Visible et l'invisible, suivi de Notes de travail. Paris: Gallimard, 1979.

Metz, Christian. "Å propos de l'impression de réalité au cinéma", Essais sur la signification au cinéma, tome I. Paris: Klincksieck (1971), p. 13-24.

Mostowicz, Ludwika. "Vers la phénoménologie sémiotique». Sémiotica, vol. 41, $\mathrm{n}^{\prime \prime \prime \prime} 1-4$ (1982), p. 41-72. 
Nordahl Lund, Steffen. "Discours et réalité ". Orbis Litterarum, vol. 47, n" 2 (1992), p. 65-80.

Ouellet, Pierre. Voir et savoir. La perception des univers du discours. Candiac: Éditions Balzac, 1992.

Ropars-Wuilleumier, Marie-Claire. L'Idée d'image. Saint-Denis: Presses Universitaires de Vincennes, 1995.

Roy, Lucie. "L'imagge au cinéma ou le corps (d)écrit". CiNéMAS, vol. 7, n" 1-2 (1996), p. 11-35.

Wittgenstein, Ludwig. Tractatus logico-philosophicus. Paris: Gallimard, 1993. 